Article

\title{
Detectability of Osseous Lesions with a Pre-Programmed Low-Dose Protocol for Cone-Beam Computed Tomography
}

\author{
Quirin Döbelin *, Bernd Stadlinger, Daniel B. Wiedemeier, Dominique Bichsel, Martin Rücker \\ and Silvio Valdec $\mathbb{D}$
}

Clinic of Cranio-Maxillofacial and Oral Surgery, Center of Dental Medicine, University of Zurich, Plattenstrasse 11, 8032 Zürich, Switzerland; bernd.stadlinger@zzm.uzh.ch (B.S.);

daniel.wiedemeier@zzm.uzh.ch (D.B.W.); dominique.bichsel@zzm.uzh.ch (D.B.); martin.ruecker@usz.ch (M.R.); silvio.valdec@zzm.uzh.ch (S.V.)

* Correspondence: quirin.doebelin@zzm.uzh.ch; Tel.: +41-(0)44-634-32-90

Received: 9 June 2020; Accepted: 17 July 2020; Published: 19 July 2020

\begin{abstract}
The present study aimed to compare the diagnostic reliability of a pre-set, manufacturer-specific, low-dose mode against a standard-dose mode in the detection of four different osseous lesions in the mandible with cone-beam computed tomography (CBCT). Four types of lesions (periapical lesion, extended periodontal gap, recession of the buccal lamella, sequestrum/fracture) were prepared on 40 pig mandibles. CBCT images were obtained from each mandible, with both the low-dose and standard-dose modes using the Orthophos SL CBCT device (Dentsply-Sirona, Bensheim, Germany). Twelve assessors quantitatively (detection of lesions) and qualitatively (assessment of detectability) evaluated the CBCT images in SIDEXIS 4 (Dentsply-Sirona) using a study-specific digital examination tool. A correct diagnosis was achieved in almost 71\% (LD: 70.8\%; SD: 70.9\%) of 1920 lesions, without a statistically significant difference between the low-dose and standard-dose mode. This finding was consistent across all four lesion types. In conclusion, while low-dose mode and standard-dose mode CBCT scans performed similarly in the detection of four prepared lesions of the mandible, the former may be a promising, user-friendly alternative method of obtaining radiation-optimized, three-dimensional images in accordance with the As Low As Diagnostically Acceptable (ALADA) principle.
\end{abstract}

Keywords: cone-beam computed tomography; radiation; low-dose protocols; dentomaxillofacial radiology; ALADA

\section{Introduction}

Low-dose protocols for cone-beam computed tomography (CBCT) have become a promising instrument for dose optimization in three-dimensional (3D) radiological diagnostics of the dentomaxillofacial region [1,2]. Since its introduction in 1998 [3], CBCT imaging has evolved into a popular radiographic modality in all fields of modern dental medicine [4]. CBCT has demonstrated advantages in the radiological diagnosis of osseous lesions-especially in the bucco-oral dimension. Dehiscence or fenestration defects and defect morphology can be detected significantly better with $3 \mathrm{D}$ imaging than with conventional radiographs [5,6]. CBCT also provides relatively more surgically relevant information than conventional two-dimensional (2D) imaging when applied to oral and maxillofacial traumatology and the assessment of bone pathologies such as osteomyelitis [7]. While superimposition-free imaging of the hard tissue of the facial skull induces less radiation relative to multi-sliced computed tomography [8,9], the applied radiation dose is still higher than that emitted by conventional 2D imaging modalities (panoramic or cephalometric radiography) $[10,11]$. 
Hence, whether the higher radiation risk justifies the diagnostic benefits of CBCT must be considered prior to its administration [12]. Further, the diagnostic reliability of radiation-optimized CBCT methods, such as low-dose protocols, warrants analysis [13].

Significant dose optimization can be achieved by limiting the field of view (FOV) to the region of interest $(\mathrm{ROI})$ and by modulating tube voltage $(\mathrm{kV})$ and tube current $(\mathrm{mA})$ [1]. However, the modulation of the scanning parameters (e.g., $\mathrm{kV}, \mathrm{mA}$ ) always depends on the CBCT scanner in use [12]. In 2013, approximately $50 \mathrm{CBCT}$ devices from 20 companies were available [14], confounding their adjustment to a standardized (dose-optimized) scanning protocol for a specific diagnostic task [15]. Furthermore, the modification of tube voltage $(\mathrm{kV})$ and tube current $(\mathrm{mA})$ and the reduction in the exposure time result in altered image contrast and image noise [12]. Nevertheless, low-dose CBCT protocols are reportedly sufficient for performing different diagnostic tasks in 3D dentomaxillofacial radiologic practice, and could constitute an important part of the dose optimization process in terms of the As Low As Diagnostically Acceptable (ALADA) principle [2,15-17].

Predefined device-specific low-dose settings can represent a simple, user-friendly possibility for predictable image quality - especially for radiographic examination, where a greater FOV is needed. The aim of this study was to evaluate the diagnostic accuracy of such a predefined device-specific, low-dose protocol compared to the standard-dose protocol. Due to ethical and safety concerns, however, pursuing these avenues of exploration cannot be performed in vivo [13]. Because the human and pig jaws feature related macroscopic anatomy and tissue composition, the pig model has been proven as a suitable and popular in vitro model in dentistry $[18,19]$. Hence, the present study induced four osseous lesions in pig mandibles to perform the aforementioned investigation.

\section{Materials and Methods}

\subsection{Preparation of Bone Defects}

The present study used 40 mandibles of pig cadavers, bought from a local butcher shop. A declaration of non-responsibility from the cantonal veterinary office was obtained from the Department of Animal Welfare and $3 R$ at the University of Zurich.

After partial soft-tissue removal, four types of osseous bone defects were prepared using drilling instruments (rose head bur H141, diamant bur 859, bur ("Lindemann") H162, Komet Dental, DENTAL Brasseler GmbH, Lemgo, Germany; handpiece Kavo Expertmatic E10C, KaVo Dental AG, Kloten, Switzerland): periapical lesion (PL), extended periodontal gap (EP), recession of the buccal layer (BL), and sequestrum/fracture (SF). All lesions within each lesion type were prepared with the same type of drilling instrument to ensure lesions were as identical as possible (PL/BL, rose head bur H141; EP, diamant bur 859; SF, “Lindemann” bur H162).

The two-rooted first premolars were separated and extracted from all pig jaws prior to the preparation of the PL. Subsequent to the drilling of the artificial apical bone defect, the roots were repositioned into the alveoles. To simulate a BL, the buccal bone layer around the cervical part of the tooth was removed. EP were dissected by drilling along the upper third of the tooth root. Cortical bone defects were created in the jaw angle to simulate SF.

Each lesion type was prepared 20 times within the 40 mandibles in a randomized order, resulting in up to four different types of lesions per mandible (Figure 1).

\section{2. $C B C T$ Examination}

The Orthophos SL 3D (Dentsply-Sirona, Bensheim, Germany) was used for the radiographic examination. All mandibles were placed and aligned centrally on a created platform by using the positioning lights of the scanner. Every mandible was scanned with the low-dose protocol $(85 \mathrm{kV}$, $13 \mathrm{~mA}$, exposure time $2.2 \mathrm{~s}$; pixel size $0.160 \mathrm{~mm}$ ) and the standard-dose protocol $(85 \mathrm{kV}, 13 \mathrm{~mA}$, exposure time $4.4 \mathrm{~s}$; pixel size $0.160 \mathrm{~mm}$ ) at a selected FOV of $11 \times 10 \mathrm{~cm}$ (Table 1). 
To mimic in vivo conditions, soft tissue was simulated with a cold-pack $(12 \times 29 \mathrm{~cm}$, GELLO Geltechnik GmbH, Ahaus, Germany) placed in the middle of the jaw.

The 80 CBCT scans (40, low-dose; 40, standard dose) were uploaded to a modified version of Sidexis 4 - Software (Dentsply Sirona, York, PA, USA) and randomly grouped into 16 datasets, each of which contained five CBCT scans.

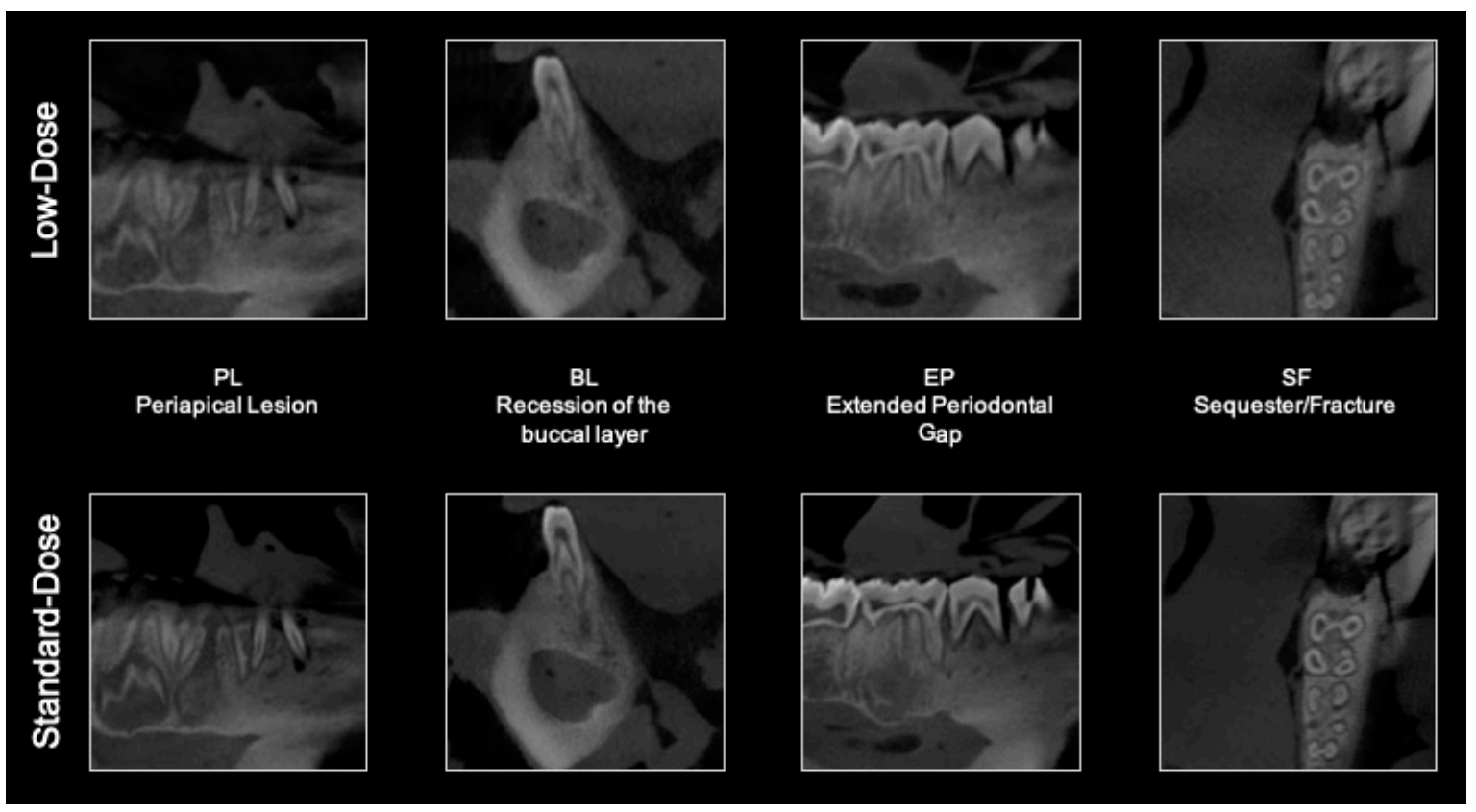

Figure 1. Screenshots of the four lesion types imaged with low-dose (upper row) and standard-dose mode (lower row) of cone-beam computed tomography (CBCT).

Table 1. Low-dose and standard-dose mode settings of the Orthophos SL (Dentsply Sirona, York, PA, USA) [20].

\begin{tabular}{cccccc}
\hline Mode & FOV $(\mathbf{c m})$ & $\mathbf{k V} / \mathbf{m A}$ & Radiation Time $(\mathbf{s})$ & Voxel Size $(\boldsymbol{\mu m})$ & Effective Dose $(\boldsymbol{\mu S v})$ \\
\hline LD & $11 \times 10$ & $85 / 13$ & 2.2 & 160 & 20 \\
SD & $11 \times 10$ & $85 / 13$ & 4.4 & 160 & 145 \\
\hline
\end{tabular}

Abbreviations: FOV, field of view.

\subsection{Image Evaluation}

All images were assessed by 12 examiners (eight oral surgeons, four maxillofacial surgeons) with at least two years of training in oral radiology and diagnosing CBCT images. All evaluators were calibrated by receiving instructions (by the primary investigator QD) during the examination of five test mandibles. Assessment was performed in three steps: the marking of a lesion with a cursor, the indication of the lesion type, and the qualitative visibility grading of the lesion: from 1 , very low to 10 , very high.

All scans were assessed in Sidexis 4 using the same workstation (Supermicro, Windows 10 Professional Edition 64 Bit; Intel ${ }^{\circledR}$ Core $^{\mathrm{TM}}$ i7-6700 CPU (C) 3.40GHz Intel64 Family 6 Model 94 Stepping 3, $3400 \mathrm{MHz} / x 64$ ) and display (HP Z23n 58, 4 cm, 23 Zoll, IPS LED Backlight).

Each mark was registered in a digital coordinate system within Sidexis 4 , and the coordinates were saved in an Excel sheet (Microsoft Excel 2016, Microsoft Corporation, Redmond, WA, USA) along with the lesion type and the qualitative visibility grading information. 


\subsection{Statistical Analysis}

Mixed-effects logistic regression was fitted to the dataset in order to estimate the differences in the detection rate between the low-dose and standard-dose protocols with respect to the four osseous lesions. The target variable (correct detection) was modelled by the fixed variables (radiation dose and lesion type) using random intercepts (pig jaw and examiner). Model diagnosis with residuals showed no severe violations of model assumptions. Thus, marginal means were computed with respect to the fixed effects, and post hoc pairwise comparisons were performed and adjusted for multiple testing according to Tukey's method. The level of significance was set to $\alpha=0.05$.

All statistical analyses and plots were performed using the statistical software R [21], including the packages lmerTest [22], emmeans [23], and DHARMa [24].

\section{Results}

The detectability rates of the four lesion types differed independently of the radiation dose (Figure 2). Nearly $71 \%$ of lesions were correctly detected (LD $=70.8 \%$; $\mathrm{SD}=70.9 \%$ ).

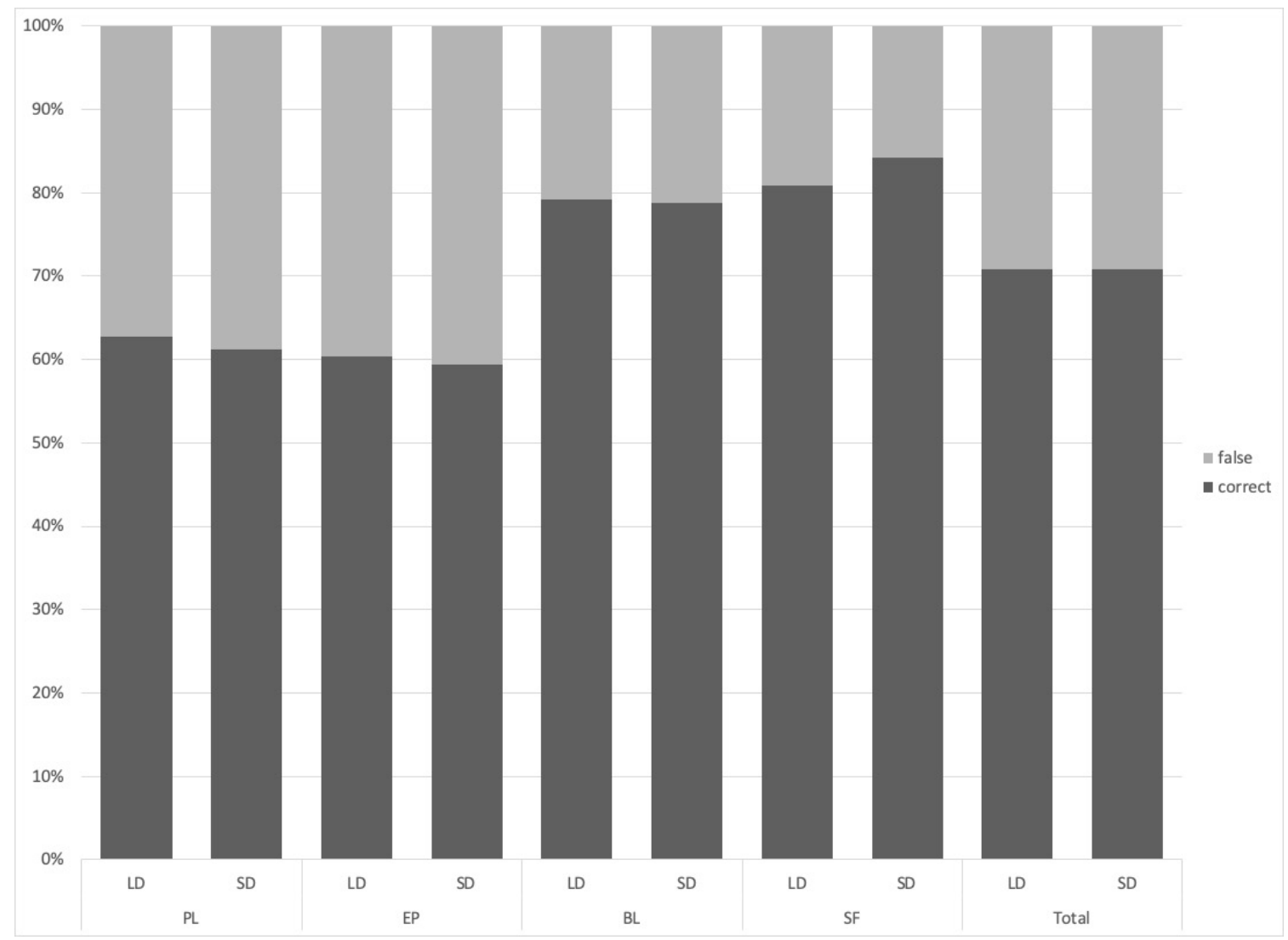

Figure 2. Detectability rates of the four different lesions (percentage).

The PL was detected correctly by the examiners in $62 \%$ of the cases (LD $=62.7 \%$; $\mathrm{SD}=61.3 \%$ ); $\mathrm{EP}, 60 \%(\mathrm{LD}=60.4 \%$; $\mathrm{SD}=59.4 \%) ; \mathrm{BL}, 79 \%(\mathrm{LD}=79.2 \%$; $\mathrm{SD}=78.8 \%)$; and $\mathrm{SF}, 83 \%(\mathrm{LD}=80.8 \%$; $\mathrm{SD}=84.2 \%)$. These results constitute descriptive values derived from a total of 480 observations per lesion type.

As calculated with the estimated marginal means method, predictive values for each of the four lesion types did not differ significantly between lesion detectability with the low-dose or standard-dose protocols $(p=0.9393)$. Differences in predictive values between the detection of the $\mathrm{BL}, \mathrm{SF}, \mathrm{PL}$, and EP differed significantly ( $p \leq 0.0001$; Table 2$)$. 
The dose-modes also differed little in terms of visibility (Figure 3): PL featured average low-dose and standard-dose visibilities of 6.9 and 7.0, respectively; EP, 7.4 and 7.5; BL, 8.5 and 8.8; and SF, 9.1 for both.

Table 2. Comparison of the detection rates of the different lesions.

\begin{tabular}{cccc}
\hline Lesions & \multicolumn{2}{c}{ Odds Ratio } & $p$-Values \\
\hline $\mathrm{PL} / \mathrm{BL}^{*}$ & 0.4026341 & 0.04344587 & $<0.0001$ \\
$\mathrm{PL} / \mathrm{EP}$ & 1.1017147 & 0.10824976 & 0.7575 \\
$\mathrm{PL} / \mathrm{SF} *$ & 0.3153958 & 0.03543323 & $<0.0001$ \\
$\mathrm{BL} / \mathrm{EP}{ }^{*}$ & 2.7362678 & 0.29447974 & $<0.0001$ \\
$\mathrm{BL} / \mathrm{SF}$ & 0.7833310 & 0.09380023 & 0.1736 \\
$\mathrm{EP} / \mathrm{SF}$ * & 0.2862772 & 0.03208903 & $<0.0001$ \\
\hline
\end{tabular}

PL and EP were detected significantly less than BL and SF (* statistically significant). Abbreviations: PL, periapical lesion; $\mathrm{BL}$, recession of the buccal layer; $\mathrm{EP}$, extended periodontal gap; and $\mathrm{SF}$, sequester/fracture.

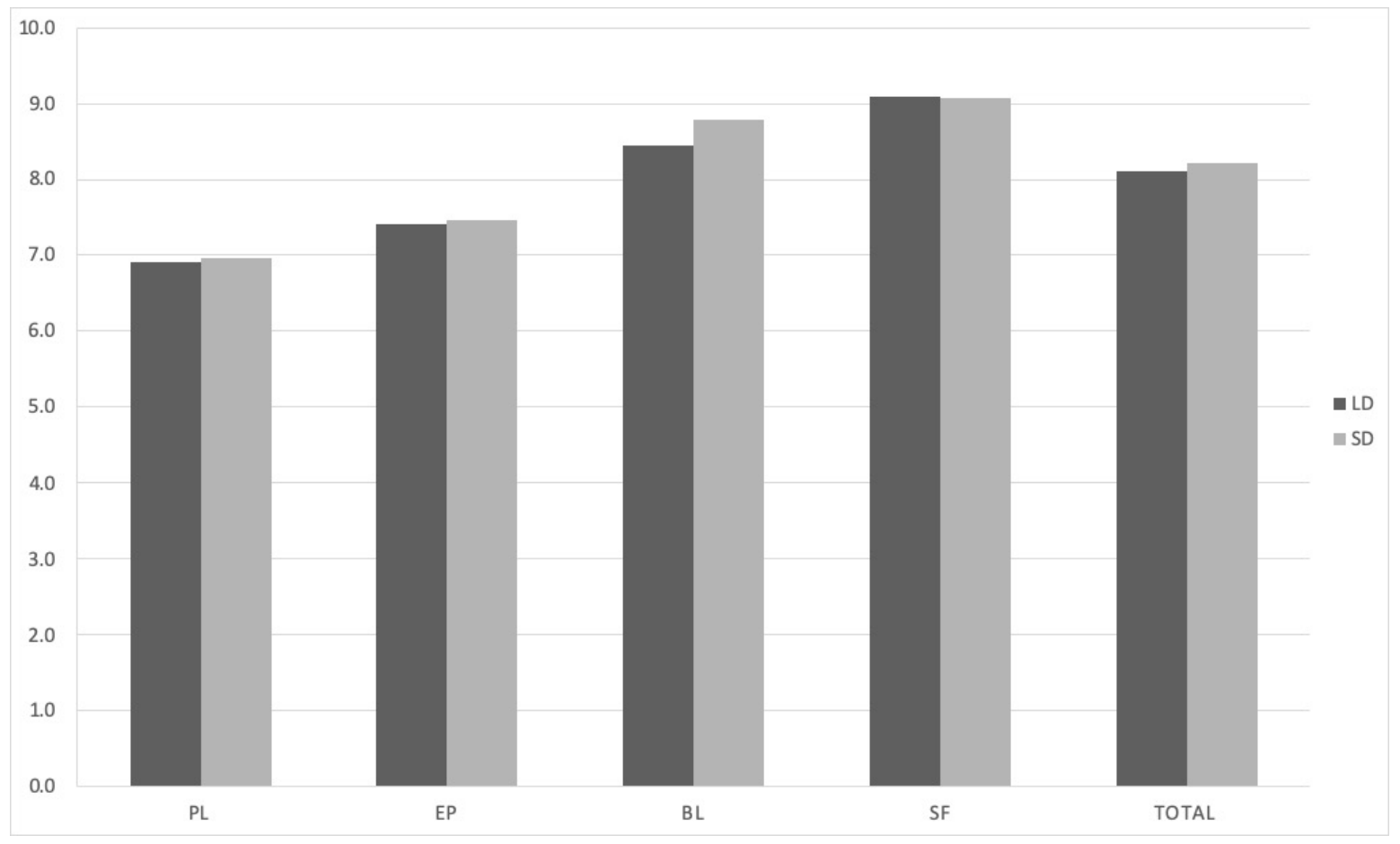

Figure 3. Assessment of the visibility of the four lesion types according to dose-mode $(1=$ very poor, $10=$ perfect). Abbreviations: PL, periapical lesion; BL, recession of the buccal layer; EP, extended periodontal gap; SF, sequestrum/fracture; LD, low dose; and SD, standard dose.

\section{Discussion}

The increasing use of CBCT in dentistry underscores the need to minimize the patients' exposure to radiation in accordance with the ALADA principle [12]. While low-dose CBCT protocols allow for minimal exposure, their diagnostic reliability relative to that of standard protocols must be determined in order to define the indication areas [13]. To investigate the diagnostic reliability of a pre-programmed, low-dose protocol with respect to typical osseous lesions, as well as fractures and sequestra, we employed an in vitro model widespread in dental medicine; the porcine jaw is well-suited to the investigation of dento-maxillofacial questions due to its resemblance to the macroscopic anatomy and composition of the human jaw $[18,19]$.

A total of 1920 lesions were evaluated in this study. On average, almost $71 \%$ of all four lesions were correctly detected with a negligible difference between the low-dose and standard-dose modes $(\mathrm{LD}=70.8 \%$ (1359); $\mathrm{SD}=70.9 \%(1361))$. Great importance was given to a cautious preparation 
technique when generating the lesions-e.g., although only 20 jaws were used to generate the PL simulations, all first premolars were extracted. By doing so, it became possible to exclude extraction signs as a possible confounder influencing the evaluation. Missing buccal lamellae were prepared on multi-rooted teeth, and fractures/sequestra were prepared in the jaw angle in the absence of alveolar foramina, which could influence the evaluation. The aim was to create a discreet finding to detect a possible difference between the applied radiation doses in small lesions. This may be an explanation for the overall detection rate of only $71 \%$. However, these results compare favorably to those of Hedesiu et al., who observed detection rates of $67.5-74.5 \%$ when examining prepared osseous lesions in pig mandibles using three different CBCT devices at a standard dose range [25].

The main objective of the present study was to ascertain possible differences in detection rates between low-dose and standard-dose protocols. This investigation failed to observe any statistically significant differences between low-dose and standard-dose CBCT protocols in the detectability of four different osseous lesions. We further found PL and EP to be correctly detected significantly less often than SF and BL; the former pair may have been rendered less visible also on account of the cautious preparation method and macroscopic anatomical differences between the pig and human jaws. Consistent with this finding, the evaluation for the visibility of the lesion types demonstrated that PL and EP were less visible than the BL and SF in both the low-dose and standard-dose modes, with little difference between the two.

The low-dose method employed in our investigation achieved an effective radiation dose reduction of $85 \%(\mathrm{LD}=20 \mathrm{mSv} ; \mathrm{SD}=145 \mathrm{mSv}[20,26])$ by halving the radiation time $(\mathrm{LD}=2.2 \mathrm{~s} ; \mathrm{SD}=4.4 \mathrm{~s})$ and using a larger copper diaphragm ( $\mathrm{LD}=1 \mathrm{~mm}$ copper diaphragm; $\mathrm{SD}=0.3 \mathrm{~mm}$ copper diaphragm). Three-dimensional information can thus be obtained by means of a conventional imaging modality like the panoramic radiograph [27].

Despite the benefit of these changes, limiting the FOV to the ROI remains a significant factor in reducing the effective radiation dose [1,9]. Pauwels et al. showed that a dose reduction of approximately $82 \%$ is possible with the selection of the smallest FOV $(4 \times 4 \mathrm{~cm})$ rather than the largest $(17 \times 12 \mathrm{~cm})$ [28]. The vertical limitation of the FOV in particular helps to protect radiation-sensitive organs such as the thyroid gland or the eye lenses and thus contributes to a reduction in the effective dose [29].

As a secondary approach for dose optimization, the modulation of tube voltage $(\mathrm{kV})$ and tube current $(\mathrm{mA})$ can reduce the dose used by standard protocols by up to $40 \%$ [1].

PL could be diagnosed much more frequently with $\mathrm{CBCT}$ than with conventional imaging methods such as panoramic and periapical radiography [30,31]. 3D imaging could be particularly relevant in cases where there is a discrepancy between the diagnostic findings of the intraoral X-ray and the clinical situation. In such cases, possible clinical causes like, e.g., untreated root canals (MB2) or root resorptions, are visualized [32]. As the FOV does not seem to have a significant impact on detectability with CBCT, it should be chosen to be as small as possible [25]. Indeed, our results indicate that a sufficient dose reduction can be achieved with the application of the low-dose protocol without any significant detriment to the detectability of periodontal lesions.

A recent review has shown that $\mathrm{CBCT}$ can visualize periodontal lesions with furcation involvement and allows for the adequate examination of periodontal defects in the bucco-oral dimension $[6,33]$. However, the application of CBCT in the diagnosis of marginal bone resorption is still a second-level imaging method, following primary 2D radiological evaluation [33]; with the application of the low-dose protocol, the clinical examination can benefit from 3D information without risking significant radiation exposure. In support of this argument, Al-Okshi et al. recently showed that dose-optimized CBCT is sufficient for the assessment of periodontal structures [15].

For the assessment of anatomical landmarks such as the buccal lamella and the maxillary sinus or the diagnosis of fractures, 3D information can be crucial for clinical decision-making. Low-dose $\mathrm{CBCT}$ has been shown to be sufficient for adequately diagnosing midfacial fractures as well as for pre-surgical implant planning [34-36]. Low-dose protocols also have potential application in the 
radiological follow-up of bone pathologies (e.g., fibro-osseous lesions), as 3D data may reveal changes earlier than 2D information collected with conventional radiography.

In addition to the aforementioned limitation of having not employed a clinical study design, the present investigation may have also been limited by our means of evaluating the CBCT datasets; as the evaluators received the same training in recognizing the four lesion types, a learning effect may have influenced our findings, leading to better diagnostics in later performed CBCT analyses. However, as the evaluators in the present study had at least two years of experience with CBCT images at the time of evaluation, this learning effect during the study should have been negligible. Concerning the expected increased use of low-dose CBCT in the future, it should be noted that the reporting of 3D datasets is more complex compared to $2 \mathrm{D}$ datasets, and thus requires specific training [37].

\section{Conclusions}

The present study demonstrated the similarity between the diagnostic reliabilities of a specific pre-programmed low-dose protocol and a standard-protocol for CBCT. However, our results cannot be applied to all low-dose protocols because of device-specific differences. As suggested in a recent narrative review, more devices need to be tested for the reliability of their low-dose protocols [13].

Author Contributions: Q.D. performed the radiological examinations, was responsible for data collection and analysis and original draft preparation; B.S., M.R., S.V. and D.B.W. reviewed and edited the manuscript. Statistical analysis were performed by D.B.W.; Conceptualization and methodology, S.V., M.R. and D.B. Preparation of the lesions was performed by S.V. and D.B.; Data validation, Q.D., D.B.W. and S.V.; Funding acquisition, M.R. and S.V. All authors have read and agreed to the published version of the manuscript.

Funding: This research was supported by Dentsply Sirona.

Acknowledgments: The authors would like to thank Dentsply Sirona for financial support. Further, we would like to thank Jan Peter Schäfer (Head of Application Software (R\&D Imaging), Dentsply Sirona) and Dietrich Roth (Product Manager Innovation, Dentsply Sirona) for their technical support as well as Franziska Riversa (Head of Product Management Extraoral Imaging, Dentsply Sirona) for assisting us during the project. The authors would also like to thank the examiners of the Center of Dental Medicine (University Zurich) and the University Hospital Zurich for the evaluation of the images.

Conflicts of Interest: The authors declare no conflict of interest. The funder had no role in the design of the study; in the collection, analyses, or interpretation of data; in the writing of the manuscript, or in the decision to publish the results.

\section{References}

1. Panmekiate, S.; Rungwittayathon, P.; Suptaweeponboon, W.; Tangtraitham, N.; Pauwels, R. Optimization of exposure parameters in dental cone beam computed tomography using a 3-step approach. Oral Surg. Oral Med. Oral Pathol. Oral Radiol. 2018, 126, 545-552. [CrossRef] [PubMed]

2. Feragalli, B.; Rampado, O.; Abate, C.; Macrì, M.; Festa, F.; Stromei, F.; Caputi, S.; Guglielmi, G. Cone beam computed tomography for dental and maxillofacial imaging: Technique improvement and low-dose protocols. Radiol. Med. 2017, 122, 581-588. [CrossRef]

3. Mozzo, P.; Procacci, C.; Tacconi, A.; Martini, P.T.; Andreis, I.A. A new volumetric CT machine for dental imaging based on the cone-beam technique: Preliminary results. Eur. Radiol. 1998, 8, 1558-1564. [CrossRef] [PubMed]

4. Bornstein, M.M.; Horner, K.; Jacobs, R. Use of cone beam computed tomography in implant dentistry: Current concepts, indications and limitations for clinical practice and research. Periodontology 2000 2017, 73, 51-72. [CrossRef]

5. Braun, X.; Ritter, L.; Jervoe-Storm, P.M.; Frentzen, M. Diagnostic accuracy of CBCT for periodontal lesions. Clin. Oral Investig. 2014, 18, 1229-1236. [CrossRef]

6. De Faria Vasconcelos, K.; Evangelista, K.M.; Rodrigues, C.D.; Estrela, C.; De Sousa, T.O.; Silva, M.A. Detection of periodontal bone loss using cone beam CT and intraoral radiography. Dentomaxillofac. Radiol. 2012, 41, 64-69. [CrossRef] [PubMed] 
7. Wolff, C.; Mucke, T.; Wagenpfeil, S.; Kanatas, A.; Bissinger, O.; Deppe, H. Do CBCT scans alter surgical treatment plans? Comparison of preoperative surgical diagnosis using panoramic versus cone-beam CT images. J. Craniomaxillofac. Surg. 2016, 44, 1700-1705. [CrossRef]

8. Kiljunen, T.; Kaasalainen, T.; Suomalainen, A.; Kortesniemi, M. Dental cone beam CT: A review. Phys. Med. 2015, 31, 844-860. [CrossRef]

9. Scarfe, W.C.; Farman, A.G. What is cone-beam CT and how does it work? Dent. Clin. N. Am. 2008, 52, 707-730. [CrossRef]

10. Bornstein, M.M.; Scarfe, W.C.; Vaughn, V.M.; Jacobs, R. Cone beam computed tomography in implant dentistry: A systematic review focusing on guidelines, indications, and radiation dose risks. Int. J. Oral Maxillofac. Implants 2014, 29, 55-77. [CrossRef]

11. Roberts, J.A.; Drage, N.A.; Davies, J.; Thomas, D.W. Effective dose from cone beam CT examinations in dentistry. Br. J. Radiol. 2009, 82, 35-40. [CrossRef] [PubMed]

12. McGuigan, M.B.; Duncan, H.F.; Horner, K. An analysis of effective dose optimization and its impact on image quality and diagnostic efficacy relating to dental cone beam computed tomography (CBCT). Swiss Dent. J. 2018, 128, 297-316. [PubMed]

13. Yeung, A.W.K.; Jacobs, R.; Bornstein, M.M. Novel low-dose protocols using cone beam computed tomography in dental medicine: A review focusing on indications, limitations, and future possibilities. Clin. Oral Investig. 2019, 23, 2573-2581. [CrossRef] [PubMed]

14. Nemtoi, A.; Czink, C.; Haba, D.; Gahleitner, A. Cone beam CT: A current overview of devices. Dentomaxillofac. Radiol. 2013, 42, 20120443. [CrossRef] [PubMed]

15. Al-Okshi, A.; Theodorakou, C.; Lindh, C. Dose optimization for assessment of periodontal structures in cone beam CT examinations. Dentomaxillofac. Radiol. 2017, 46, 20160311. [CrossRef] [PubMed]

16. Jaju, P.P.; Jaju, S.P. Cone-beam computed tomography: Time to move from ALARA to ALADA. Imaging Sci. Dent. 2015, 45, 263-265. [CrossRef] [PubMed]

17. White, S.C.; Scarfe, W.C.; Schulze, R.K.W.; Lurie, A.G.; Douglass, J.M.; Farman, A.G.; Law, C.S.; Levin, M.D.; Sauer, R.A.; Valachovic, R.W.; et al. The Image Gently in Dentistry campaign: Promotion of responsible use of maxillofacial radiology in dentistry for children. Oral Surg. Oral Med. Oral Pathol. Oral Radiol. 2014, 118, 257-261. [CrossRef]

18. Wang, S.; Liu, Y.; Fang, D.; Shi, S. The miniature pig: A useful large animal model for dental and orofacial research. Oral Dis. 2007, 13, 530-537. [CrossRef]

19. Kantarci, A.; Hasturk, H.; Van Dyke, T.E. Animal models for periodontal regeneration and peri-implant responses. Periodontology 2000 2015, 68, 66-82. [CrossRef]

20. Dentsply Sirona. Orthophos SL: Instruction manual; Dentsply Sirona: Bensheim, Germany, 2018.

21. R Core Team. R: A Language and Environment for Statistical Computing [Computer Program]; R Foundation for Statistical Computing: Vienna, Austria, 2015. Available online: https://www.R-project.org/. (accessed on 9 June 2020).

22. Kuznetsova, A.; Brockhoff, P.B.; Christensen, R.H.B. lmerTest Package: Tests in Linear Mixed EffectsModels. J. Stat. Softw. 2017, 82, 1-26. [CrossRef]

23. Lenth, R.; Emmeans: Estimated Marginal Means, aka Least-Squares Means [Computer Program]. R Package Version 1.2.3. 2018. Available online: https://CRAN.R-project.org/package=emmeans (accessed on 9 June 2020).

24. Hartig, F.; DHARMa: Residual Diagnostics for Hierarchical (Multi-Level/Mixed) Regression Models [Computer Program]. R Package Version 0.2.4. 2019. Available online: https://CRAN.R-project.org/package= DHARMa (accessed on 9 June 2020).

25. Hedesiu, M.; Baciut, M.; Baciut, G.; Nackaerts, O.; Jacobs, R. Consortium, S. Comparison of cone beam CT device and field of view for the detection of simulated periapical bone lesions. Dentomaxillofac. Radiol. 2012, 41, 548-552. [CrossRef]

26. Ludlow, J.B. Dosimetry of Orthophos SL CBCT Unit with Low-Dose Protocol; North Carolina Oral Health Institute: Chapel Hill, NC, USA, 2014; unpublished.

27. Visser, H.; Hermann, K.P.; Bredemeier, S.; Kohler, B. Dose measurements comparing conventional and digital panoramic radiography. Mund Kiefer Gesichtschir 2000, 4, 213-216. [CrossRef] [PubMed] 
28. Pauwels, R.; Zhang, G.; Theodorakou, C.; Walker, A.; Bosmans, H.; Jacobs, R.; Bogaerts, R.; Horner, K. Effective radiation dose and eye lens dose in dental cone beam CT: Effect of field of view and angle of rotation. Br. J. Radiol. 2014, 87, 20130654. [CrossRef]

29. Ludlow, J.B.; Timothy, R.; Walker, C.; Hunter, R.; Benavides, E.; Samuelson, D.B.; Scheske, M.J. Effective dose of dental CBCT-a meta analysis of published data and additional data for nine CBCT units. Dentomaxillofac. Radiol. 2015, 44, 20140197. [CrossRef] [PubMed]

30. Estrela, C.; Bueno, M.R.; Leles, C.R.; Azevedo, B.; Azevedo, J.R. Accuracy of cone beam computed tomography and panoramic and periapical radiography for detection of apical periodontitis. J. Endod. 2008, 34, 273-279. [CrossRef]

31. Bornstein, M.M.; Lauber, R.; Sendi, P.; Von Arx, T. Comparison of periapical radiography and limited cone-beam computed tomography in mandibular molars for analysis of anatomical landmarks before apical surgery. J. Endod. 2011, 37, 151-157. [CrossRef] [PubMed]

32. Lo Giudice, R.; Nicita, F.; Puleio, F.; Alibrandi, A.; Cervino, G.; Lizio, A.S.; Pantaleo, G. Accuracy of periapical radiography and CBCT in endodontic evaluation. Int. J. Dent. 2018, 2018, 2514243. [CrossRef]

33. Haas, L.F.; Zimmermann, G.S.; De Luca Canto, G.; Flores-Mir, C.; Correa, M. Precision of cone beam CT to assess periodontal bone defects: A systematic review and meta-analysis. Dentomaxillofac. Radiol. 2018, 47, 20170084. [CrossRef]

34. Rozema, R.; Doff, M.H.; Van Ooijen, P.M.; Postmus, D.; Westerlaan, H.E.; Boomsma, M.F.; Van Minnen, B. Diagnostic reliability of low dose multidetector CT and cone beam CT in maxillofacial trauma-an experimental blinded and randomized study. Dentomaxillofac. Radiol. 2018, 47, 20170423. [CrossRef]

35. El Sahili, N.; David-Tchouda, S.; Thoret, S.; Nasseh, I.; Berberi, A.; Fortin, T. Effect of Milliamperage Reduction on Pre-surgical Implant Planning Using Cone Beam Computed Tomography by Surgeons of Varying Experience. J. Maxillofac. Oral Surg. 2018, 17, 520-530. [CrossRef]

36. Dawood, A.; Brown, J.; Sauret-Jackson, V.; Purkayastha, S. Optimization of cone beam CT exposure for pre-surgical evaluation of the implant site. Dentomaxillofac. Radiol. 2012, 41, 70-74. [CrossRef] [PubMed]

37. Brown, J.; Jacobs, R.; Jaghagen, E.L.; Lindh, C.; Baksi, G.; Schulze, D.; Schulze, R. Basic training requirements for the use of dental CBCT by dentists: A position paper prepared by the European Academy of DentoMaxilloFacial Radiology. Dentomaxillofac. Radiol. 2014, 43, 20130291. [CrossRef] [PubMed] 